\title{
Facile Synthesis of Ni/Co (Hydr)oxides with Nanosheet Structure for High Performance Supercapacitors
}

\author{
Chengyu Ma ${ }^{1}$, Saisai Jian ${ }^{1}$, Jinli Qiao ${ }^{1, *}$, Joey Jung ${ }^{2, *}$ \\ ${ }^{1}$ College of Environmental Science and Engineering, Donghua University, 2999 Ren'min North Road, \\ Shanghai 201620, P. R. China \\ ${ }^{2}$ Wattech Power Inc, 150-11120 Bridgeport Rd. Richmond BC V6X1T2, Canada \\ *E-mail: qiaojl@dhu.edu.cn, jung@wattechpower.ca
}

doi: $10.20964 / 2016.12 .38$

Received: 28 August 2016 / Accepted: 2 October 2016 / Published: 10 November 2016

\begin{abstract}
In this article, the novel kind of binary metal oxides, Ni/Co (hydr)oxides, with nanosheet structure have been successfully established via a facile hydrothermal self-assembled process with ammonia solution as the reaction reagent. The as-prepared samples with peculiar morphologies and characteristics are easily realized by just controlling the calcination temperatures. The composition, morphology, and microstructure of the products were characterized by X-ray diffraction (XRD), scanning electron microscopy (SEM), high-resolution transmission electron microscopy (HR-TEM) and X-ray photoelectron spectroscopy (XPS). The thermogravimetric analysis (TGA) revealed the decomposition details of the precursor. Electrochemical measurements show that the sample calcined at $250^{\circ} \mathrm{C}$ has the optimized capacitive performance, demonstrated by cyclic voltammetry and galvanostatic charge-discharge cycling techniques. The Ni/Co hydroxide nanosheets as electrode materials for supercapacitor exhibit high specific capacitance of $1427 \mathrm{~F} \mathrm{~g}^{-1}$ at $1 \mathrm{~A} \mathrm{~g}^{-1}$, and $1270 \mathrm{~F} \mathrm{~g}^{-1}$ at $10 \mathrm{~A} \mathrm{~g}^{-1}$, indicating an excellent rate capability. Also, the superior cyclic stability with the capacitance retention of $92.3 \%$ is achieved even over 3000 cycles at a high current density of $10 \mathrm{~A} \mathrm{~g}^{-1}$. The improved capacity and cycling stability makes it promising electrode material for offering an effective way to achieve high supercapacitor performance.
\end{abstract}

Keywords: Ni/Co (hydr)oxide; Morphology-control; Calcination temperature; Supercapacitor; Cyclic stability

\section{FULL TEXT}

(C) 2016 The Authors. Published by ESG (www.electrochemsci.org). This article is an open access article distributed under the terms and conditions of the Creative Commons Attribution license (http://creativecommons.org/licenses/by/4.0/). 